\title{
Estilos de aprendizaje en los estudiantes de Educación Física y Ciencias del Deporte de la Universidad Autónoma de Querétaro
}

Learning styles in the students of Physical Education and Sports Science of the Universidad Autónoma de Querétaro

Estilos de aprendizagem em estudantes de Educação Física e Ciências do Esporte da Universidade Autônoma de Querétaro

Óscar Ángel Gómez-Terán

Universidad Autónoma de Querétaro, México oagomter@gmail.com https://orcid.org/0000-0001-6637-5217

Beatriz Garza-González Universidad Autónoma de Querétaro, México bgarza62@gmail.com http://orcid.org/0000-0002-5696-6870

Ma. Alejandra Hernández-Castañón Universidad Autónoma de Querétaro, México alehdez983@yahoo.com.mx http://orcid.org/0000-0002-9041-077X

Victorina Castrejón-Reyes Universidad Autónoma de Querétaro, México vica_60@hotmail.com http://orcid.org/0000-0002-0122-2620

Sandra Jenny Cortés-Heredia Universidad Autónoma de Querétaro, México jennycortes2000@hotmail.com https://orcid.org/0000-0001-6432-8708 


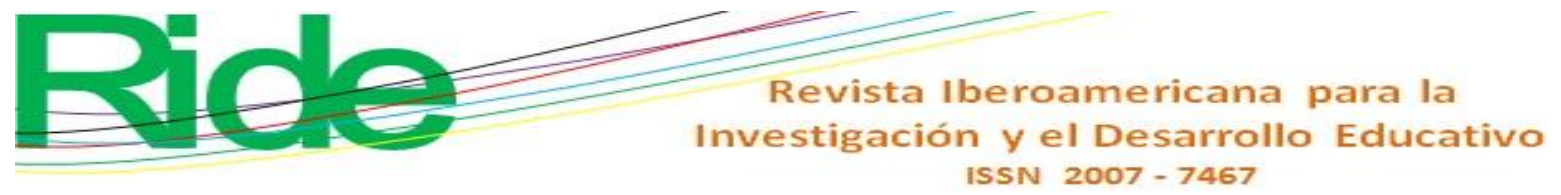

\section{Resumen}

Los estilos de aprendizaje son métodos que se emplean con el fin de organizar las cogniciones sobre el mundo para comprenderlo mejor. Un estilo no es una aptitud, sino una forma de pensar y una manera preferida de emplear las aptitudes que se poseen. Con estos antecedentes, Alonso y Honey refieren cuatro estilos básicos de aprendizaje: activo, reflexivo, teórico y pragmático. Explicado esto, el principal objetivo de este trabajo fue documentar el estilo de aprendizaje preferente en estudiantes de la licenciatura en Educación Física y Ciencias del Deporte de la Universidad Autónoma de Querétaro. El estudio fue no experimental, descriptivo-transversal, y se incluyó a la totalidad de estudiantes de la referida carrera. Para la recolección de datos se utilizó el Cuestionario Honey-Alonso de estilos de aprendizaje que consta de 20 reactivos para cada uno de los cuatro estilos de aprendizaje.

Se excluyeron de la investigación a estudiantes que no asistieron el día de la recolección de datos o que no aceptaron participar. Asimismo, se eliminaron los cuestionarios no respondidos en su totalidad. Para realizar el análisis estadístico descriptivo se utilizó el programa Statistical Package for the Social Sciences (versión 20) y se emplearon los algoritmos de Honey-Alonso para establecer el tipo de estilo predominante. Al momento de la aplicación del instrumento se explicó a los estudiantes los objetivos de la indagación y se solicitó su consentimiento verbal y su participación voluntaria. Se observó que el estilo de aprendizaje activo tuvo una preferencia entre alta y muy alta (62.75\%); sin embargo, la preferencia en el estilo reflexivo fue baja o muy baja (52.94\%). En relación con la preferencia para los estilos pragmático y teórico, esta fue moderada (55.56 \% y $52.94 \%$, respectivamente). El estilo de aprendizaje que predominó fue el activo con $67.32 \%$, situación que soslaya el estilo de aprendizaje reflexivo que, según Honey y Alonso, es el preferente en carreras del área de humanidades.

Palabras clave: CHAEA, docencia, estilos de aprendizaje, estudiante universitario. 


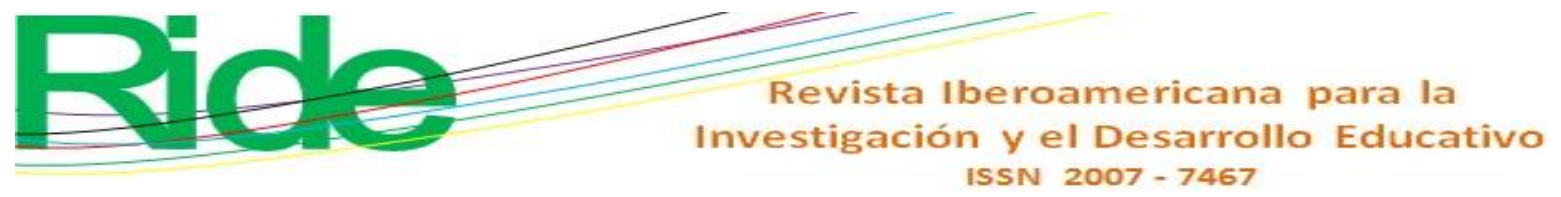

\section{Abstract}

Learning styles are methods that are used to organize cognitions about the world, to better understand it. A style is not an aptitude, but a way, but a way of thinking and preferred way of using the skill that are possessed. With this antecedents, Alonso and Honey refer four basic styles of learning: Active, Reflective, Theoretical and Pragmatic. Explained this, the main objective of this work was to document the style of preferential learning in students of Physical Education and Sports Science of the Universidad Autónoma de Querétaro. The study was Non-experimental, descriptive-transversal, and all the students of the aforementioned career were included. For data collection, the "Honey-Alonso Learning Styles Questionnaire" was used, consisting of 20 items for each of the four learning styles. Students who did not attend the data collection day or who did not accept participation were excluded from of the investigation, the questionnaires not answered in their entirety were eliminated. To perform the descriptive statistical analysis the Statistical Package for the Social Sciences program (version 20) will be established and the Honey- Alonso algorithms will be used to establish the predominant type of style. At the time of the application of the instrument, the students were explained the objectives of the inquiry and verbal consent and voluntary participation were requested. It was observed that the active learning style had a preference between high and very high $(62.75 \%)$; however, the preference in the reflective style was low or very low $(52.94 \%)$. In relation to the preference for the pragmatic and theoretical styles, this was moderate $(55.56 \%$ and $52.94 \%$ respectively). The predominant learning style was active with $67.32 \%$, a situation that ignores the reflective learning style that according to Honey and Alonso is the preferred one in careers in the humanities area.

Keywords: CHAEA, teaching, learning styles, university student. 


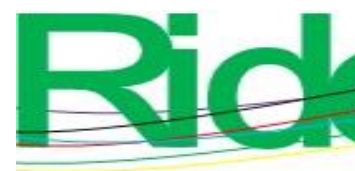

\section{Resumo}

Estilos de aprendizagem são métodos usados para organizar cognições sobre o mundo para melhor entendê-lo. Um estilo não é uma aptidão, mas uma maneira de pensar e uma maneira preferida de usar as habilidades que você possui. Com esse pano de fundo, Alonso e Honey se referem a quatro estilos básicos de aprendizado: ativo, reflexivo, teórico e pragmático. Explicou isso, o principal objetivo deste trabalho foi documentar o estilo de aprendizagem preferido em estudantes da graduação em Educação Física e Ciências do Esporte da Universidade Autônoma de Querétaro. O estudo foi não experimental, de corte transversal descritivo, e todos os alunos da carreira mencionada foram incluídos. Para a coleta de dados, foi utilizado o Questionário Honey-Alonso sobre estilos de aprendizagem, composto por 20 itens para cada um dos quatro estilos de aprendizagem.

Os alunos que não compareceram ao dia da coleta de dados ou que não concordaram em participar foram excluídos da investigação. Da mesma forma, os questionários não respondidos na íntegra foram eliminados. Para a análise estatística descritiva, foi utilizado o programa Statistical Package for the Social Sciences (versão 20) e os algoritmos HoneyAlonso para estabelecer o tipo de estilo predominante. No momento da aplicação do instrumento, os objetivos da investigação foram explicados aos alunos e seu consentimento verbal e participação voluntária foram solicitados. Observou-se que o estilo de aprendizagem ativo apresentou preferência entre alta e muito alta $(62,75 \%)$; no entanto, a preferência no estilo reflexivo foi baixa ou muito baixa $(52,94 \%)$. Em relação à preferência pelos estilos pragmático e teórico, este foi moderado (55,56\% e 52,94\%, respectivamente). O estilo de aprendizagem predominante foi ativo com $67,32 \%$, situação que evita o estilo reflexivo de aprendizagem que, segundo Honey e Alonso, é preferido nas carreiras na área de humanidades.

Palavras-chave: CHAEA, ensino, estilos de aprendizagem, estudante universitário.

Fecha Recepción: Junio 2019

Fecha Aceptación: Diciembre 2019 


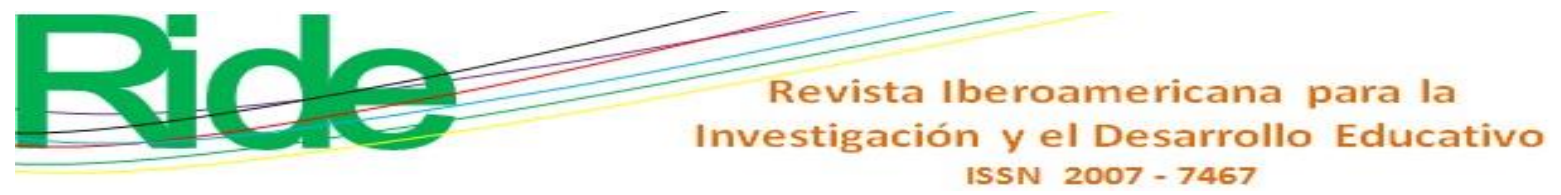

Introducción

De acuerdo con Sternberg (1997), los estilos de aprendizaje (EA) históricamente se han definido como los métodos que se emplean con el fin de organizar nuestras cogniciones sobre el mundo para comprenderlo mejor. Un estilo es una forma de pensar y una manera preferida de aprovechar las aptitudes que se poseen; esto quiere decir que la aptitud se refiere al modo en que alguien sabe hacer una cosa, mientras que el estilo tiene que ver con la manera en que a ese alguien le gusta hacer algo.

Este concepto retoma la propuesta de Dunn, Dunn y Price (1985) y Keefe (1988), quienes afirman que los EA se vinculan con la personalidad del discente; por ejemplo, si se compara a estudiantes extrovertidos frente a estudiantes introvertidos, los primeros adquieren mejor las habilidades comunicativas interpersonales, mientras que los segundos desarrollan más las habilidades lingüísticas académicas.

Para Alonso, Gallego y Honey (2007) existen cuatro estilos básicos de aprendizaje: activo, reflexivo, teórico y pragmático. Las características de las personas de acuerdo con el estilo de preferencia se describen de forma sucinta en la tabla 1. 


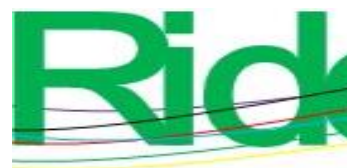

Revista Iberoamericana para la

Investigación y el Desarrollo Educativo

ISSN $2007-7467$

Tabla 1. Características de las personas según el estilo de aprendizaje preferente

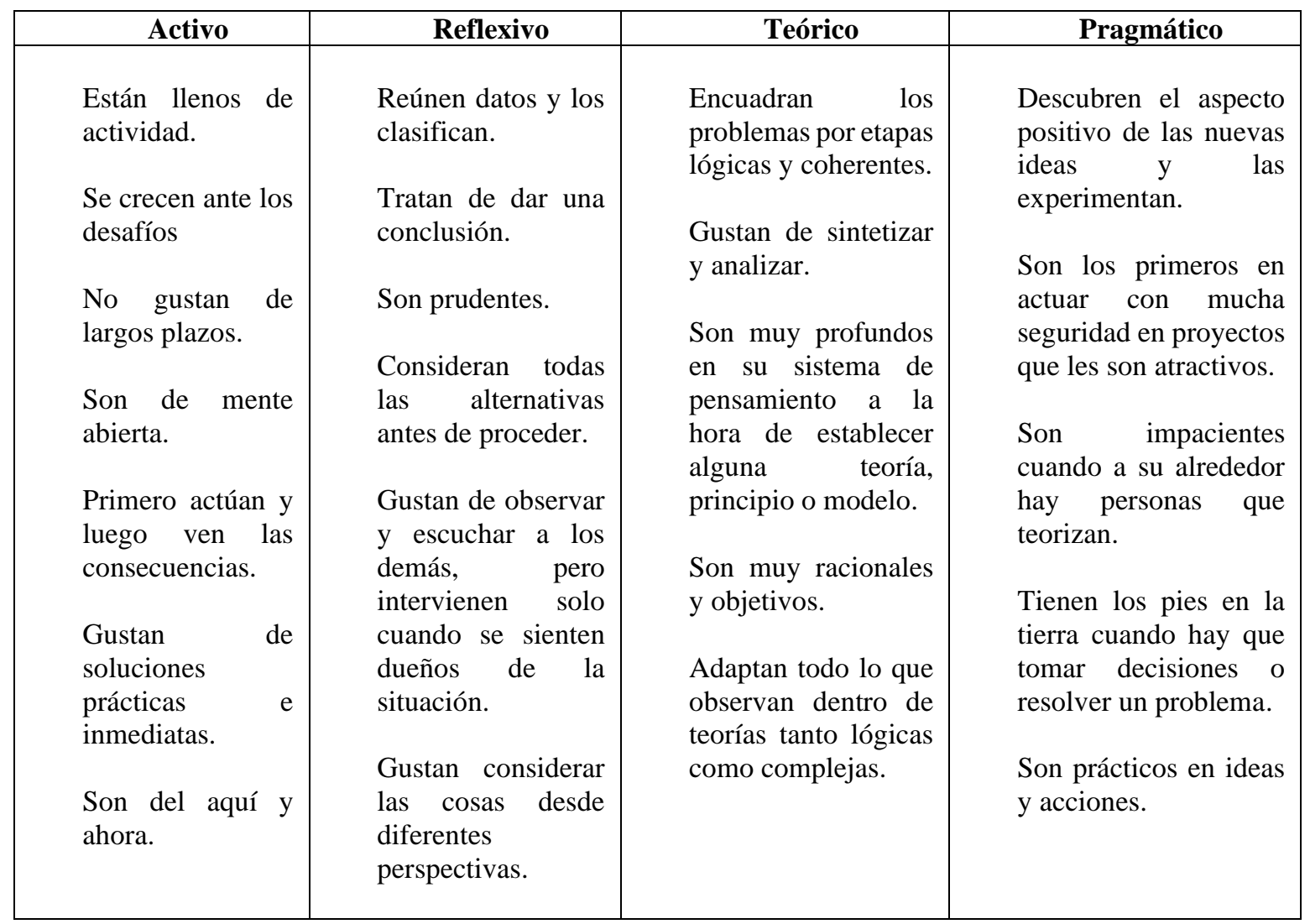

Fuente: Elaboración propia a partir de Alonso, Gallego y Honey (2007)

Continuando con Alonso et al. (2007), es elemental mencionar que esta clasificación no se relaciona directamente con la inteligencia, ya que siempre hay personas con esta facultad indistintamente de su estilo de aprendizaje predominante. Al respecto, los citados autores explican: "Parece útil la estrategia de prescindir parcialmente de la insistencia en el factor inteligencia, que no es fácil de modificar, e insistir en otras facetas del aprendizaje que sí son accesibles y mejorables" (Alonso et al., 2007, p. 70).

Aun así, y a partir de la clasificación de Alonso et al. (2007), se han realizado diferentes estudios en los que se muestra en términos generales que el estilo de aprendizaje que se destaca en estudiantes universitarios es el reflexivo, independientemente de la disciplina (Acevedo, Cavadla y Alvis, 2015; Camarero, Del Buey y Herrero, 2000).

Ahora bien, en cuanto a los antecedentes que se pueden mencionar sobre el estudio de los estilos de aprendizaje, vale acotar la investigación de Serra-Olivares, Muñoz, Cejudo y Gil (2017) realizada con estudiantes universitarios de Educación Física de la Universidad Católica de Temuco (Chile) (lapso 2015-2016). En dicho trabajo se aplicó el cuestionario 


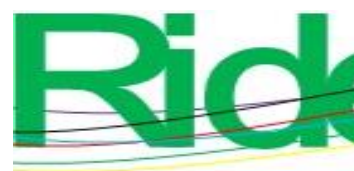

Revista Iberoamericana para la Investigación y el Desarrollo Educativo ISSN $2007-7467$

CHAEA, mediante el cual se determinó que en los participantes predominaban principalmente los estilos de aprendizaje combinado (56\%) y activo (21\%). Además, los estudiantes con un estilo de aprendizaje activo demostraron un rendimiento académico significativamente inferior al de sus compañeros que tenían un estilo de aprendizaje pragmático y combinado.

En otra indagación similar, desarrollada en España, Gil et al. (2007) evaluaron los estilos de aprendizaje de estudiantes de magisterio en la especialidad de Educación Física. El objetivo era intervenir en la implementación de los planes y programas de estudio que contribuyeran a la titulación de los estudiantes; en dicho trabajo se encontró que el estilo de aprendizaje predominante era el reflexivo (44\%), seguido del activo (23\%), el teórico (19.01 $\%)$ y el pragmático $(13 \%)$.

Asimismo, en el trabajo de Salas-Cabrera (2014), efectuado en la Universidad Nacional en Costa Rica sobre los estilos de aprendizaje predominantes en alumnos de la Escuela de Ciencias del Movimiento Humano y Calidad de Vida, se encontró que en la mayoría de los estudiantes sobresalía un estilo de aprendizaje reflexivo.

En concordancia con lo anterior, Acevedo et al. (2015) llevaron a cabo una investigación con estudiantes de la Facultad de Ingeniería de la Universidad de Cartagena Colombia. Los resultados de este trabajo demostraron que el estilo de aprendizaje predominante era el reflexivo, con $31.9 \%$ en hombres y $36.1 \%$ en mujeres.

De igual forma, en un artículo de Isaza Valencia (2014) desarrollado en la Corporación Universitaria Minuto de Dios (seccional Bello), se determinó que resaltaban los estilos pragmático y teórico sobre otros como el activo o el reflexivo. Estos hallazgos contrastan con planteado en algunos modelos pedagógicos de educación superior, pues se esperaría que los últimos deberían de ser predominantes en los estudiantes.

\section{Objetivo}

El objetivo de la presente investigación fue documentar el estilo de aprendizaje que predomina en los estudiantes de la licenciatura en Educación Física y Ciencias del Deporte de la Universidad Autónoma de Querétaro. 


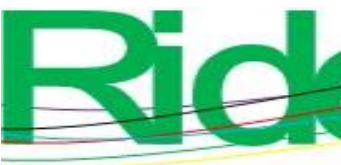

Revista Iberoamericana para la Investigación y el Desarrollo Educativo

ISSN $2007-7467$

Ahora bien, tomando como referencia lo sugerido en diferentes estudios en torno a que el estilo de aprendizaje más habitual en universitarios es el reflexivo independientemente de la especialidad cursada - , en este trabajo se ha planteado la siguiente hipótesis:

Hi. El estilo de aprendizaje que predomina en los estudiantes de la licenciatura en Educación Física y Ciencias del Deportes es el reflexivo-teórico.

\section{Metodología}

Se realizó un estudio no experimental de tipo descriptivo y transversal en el que se incluyó al total de estudiantes de la licenciatura en Educación Física y Ciencias del Deporte (153) a quienes se les aplicó una cédula de identificación sociodemográfica, además del Cuestionario Honey-Alonso de estilos de aprendizaje (CHAEA) (1986), del cual se tiene autorización para su uso por parte de los autores.

El cuestionario consta de 80 reactivos, distribuidos equitativamente (20) para cada uno de los cuatro estilos de aprendizaje referidos. Vale acotar que no se incluyó a los estudiantes que no asistieron el día de la aplicación del cuestionario o que no aceptaron participar; además, se eliminaron los cuestionarios no contestados en su totalidad. Para realizar el trabajo de campo se contó con la aprobación del Comité de Investigación de la Facultad de Enfermería de la Universidad Autónoma de Querétaro. Además, de forma conjunta con el coordinador de la licenciatura, se agendó fecha para la aplicación del cuestionario en el aula y dentro del horario escolar.

El cuestionario fue autoadministrado, para lo cual se verificó la comprensión de las indicaciones y se validó el llenado de los cuestionarios. También se solicitó a los estudiantes que al finalizar su requisición realizaran el ejercicio de ubicar su estilo de aprendizaje preferente y se ofreció una breve interpretación del resultado. Para el análisis estadístico descriptivo se utilizó el programa SPSS (versión 20). Al momento de la aplicación del instrumento, se explicaron los objetivos del estudio y se solicitó de forma verbal su consentimiento y participación voluntaria. 


\section{Resultados}

Participaron 153 estudiantes de la licenciatura en Educación Física y Ciencias del Deporte, de los cuales se exponen los siguientes resultados al aplicar el CHAEA. En la tabla 2 se observa que predomina la participación de los estudiantes del sexo masculino (74.3\%); además, según la distribución por semestre, los mayores porcentajes fueron para el segundo (25\%), octavo $(13.2 \%)$ y séptimo $(12.5 \%)$.

Tabla 2. Frecuencia de participantes según sexo y semestre cursado

\begin{tabular}{|l|l|c|c|}
\hline \multicolumn{2}{|c|}{ Variable } & Frecuencia & \% \\
\hline Sexo & Femenino & 37 & 74.3 \\
\hline & Masculino & 107 & 8.6 \\
\hline Semestre & 1 & 13 & 25.7 \\
\hline & 2 & 39 & 11.2 \\
\hline & 3 & 17 & 11.8 \\
\hline & 4 & 18 & 7.9 \\
\hline & 5 & 12 & 9.2 \\
\hline & 6 & 14 & 12.5 \\
\hline & 7 & 19 & 13.2 \\
\hline & 8 & 20 & 7 \\
\hline
\end{tabular}

Fuente: Cédula de identificación $n=153$

En la figura 1, se observa que el estilo de aprendizaje activo es el que predomina entre los participantes, con preferencia "alta y muy alta" (67.32 \%), en tanto que el estilo de aprendizaje reflexivo tiene una incidencia "baja y muy baja" (88.89 \%), resultado que es contrario a la hipótesis de investigación planteada. Por otra parte, los estudiantes tuvieron una preferencia moderada para los estilos pragmático (55.56\%) y teórico (52.94\%), situación que contrasta con los hallazgos revisados en la literatura disponible que incluyó a estudiantes de Educación Física, en donde se muestra que el estilo que sobresale es el combinado, es decir, reflexivo-teórico y activo-teórico-pragmático (56\%), seguido de los estilos activo (21\%), teórico (13\%) y pragmático (4\%) (Serra-Olivares et al., 2017). 


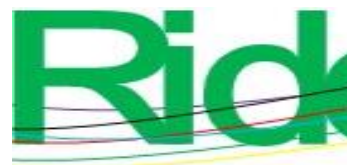

Revista Iberoamericana para la Investigación y el Desarrollo Educativo

ISSN $2007-7467$

Figura 1. Nivel de preferencia según estilo de aprendizaje en estudiantes de la licenciatura en Educación Física y Ciencias del Deporte

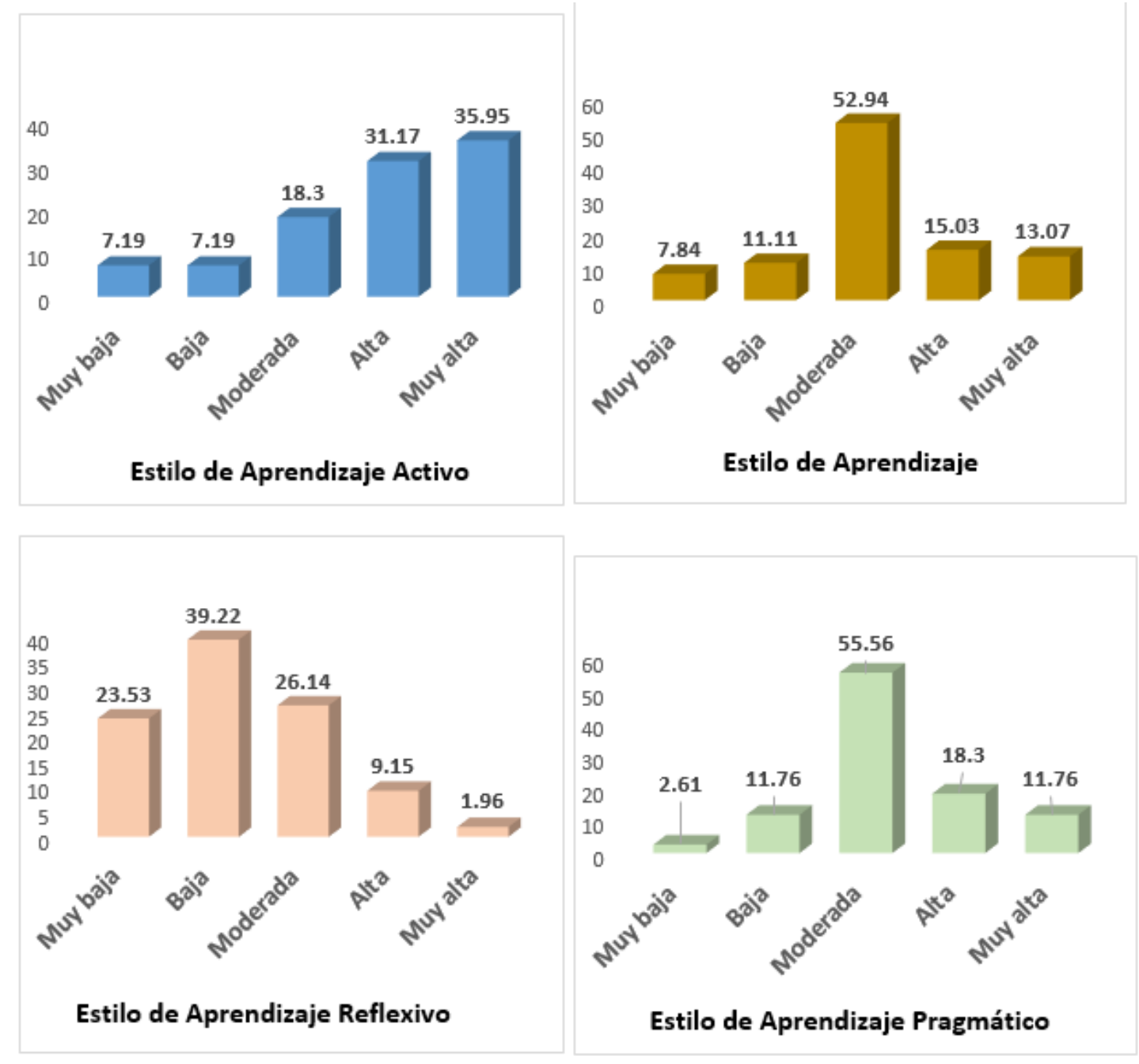

Fuente: Elaboración propia

Ahora bien, al comparar los baremos de interpretación de los cuatro estilos de aprendizaje de Alonso et al. (2007) para carreras del área de humanidades con los datos obtenidos en el presente estudio, se puede indicar que el estilo activo es el de mayor preferencia entre los estudiantes (con una media de 11.99), en tanto que el estilo reflexivo es el menos preferido (con una media de 13.34), lo que significa una diferencia positiva de 2.06 puntos para el primer estilo, pero negativa en 2.58 puntos para el segundo. Respecto a los estilos teórico y práctico, sus medias estadísticas son mayores a las propuestas por los autores citados (figura 2). 


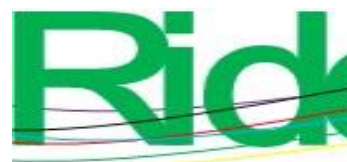

Revista Iberoamericana para la Investigación y el Desarrollo Educativo ISSN $2007-7467$

Figura 2. Media de estilo de aprendizaje preferente en estudiantes de Educación Física y Ciencias del Deporte en comparación con la propuesta de Honey et al. (2007) para carreras de humanidades

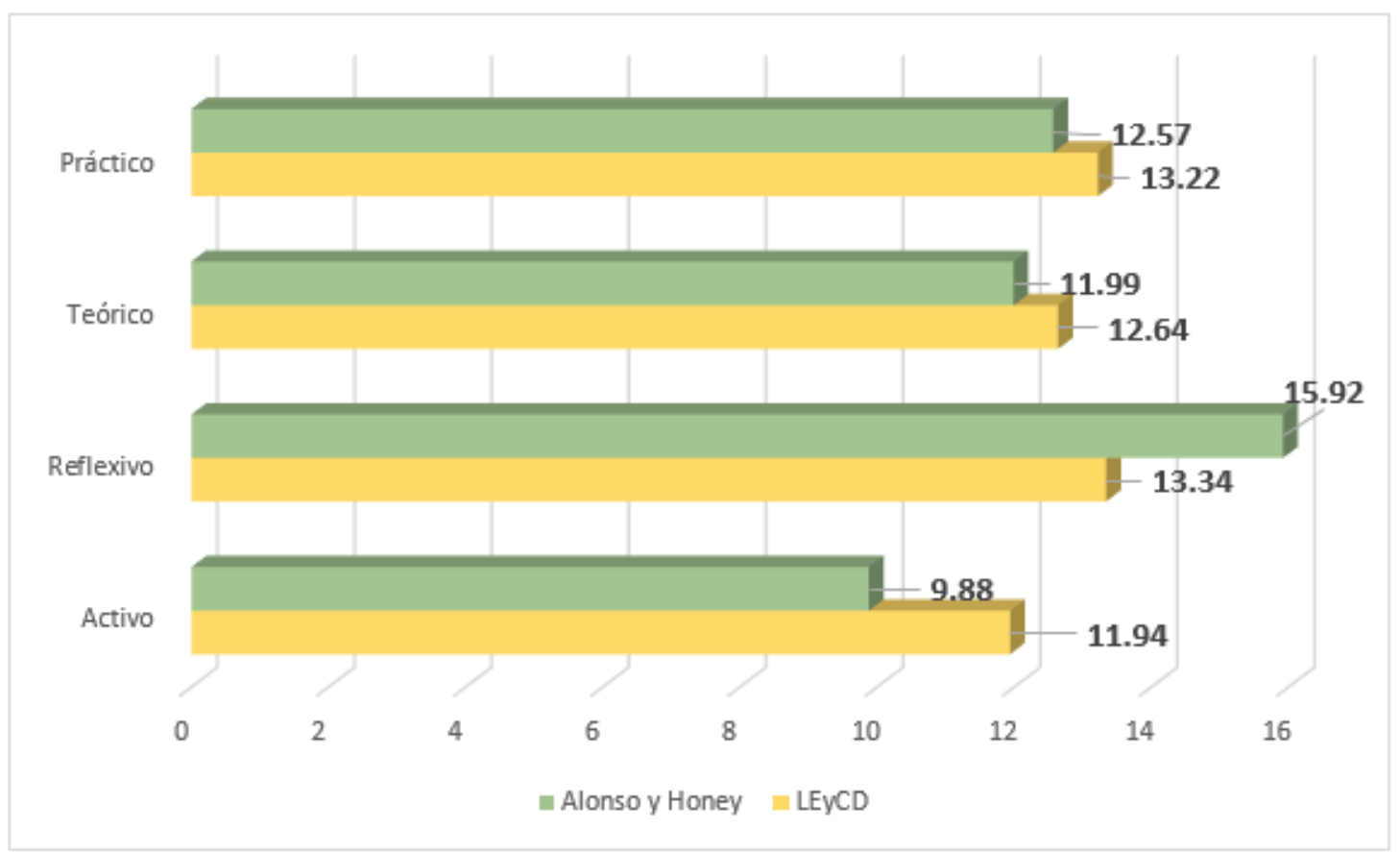

Fuente: Elaboración propia

Los datos recabados en el estudio sobre estilo de aprendizaje en estudiantes de la licenciatura en Educación Física y Ciencias del Deporte muestran una preferencia alta y muy alta para el estilo activo, moderada para los estilos teórico y pragmático, y baja para el estilo reflexivo. En otras palabras, se puede indicar que en los estudiantes se tiene la presencia de estilos de aprendizaje combinados (activo-pragmático y activo-teórico), alejándose del estilo reflexivo, situación que difiere de la mayoría de la literatura revisada que incluyó a universitarios de educación física, en los cuales predomina el estilo reflexivo (Alonso et al., 2007; Gil et al., 2007; Serra-Olivares et al., 2017). 


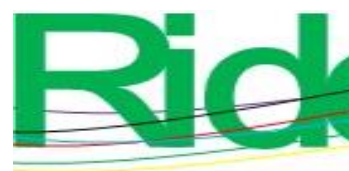

Revista Iberoamericana para la

Investigación y el Desarrollo Educativo

ISSN 2007-7467

\section{Discusión}

Con base en los resultados de investigación, se puede afirmar que entre los estudiantes de la licenciatura en Educación Física y Ciencias del Deporte sobresale el estilo de aprendizaje activo, hallazgo contrario a la hipótesis de investigación planteada, en la cual se refería que el estilo de aprendizaje de estos alumnos era reflexivo. De hecho, en este trabajo se ha demostrado que los participantes de esta investigación presentaron una preferencia moderada para los estilos pragmático $(55.56 \%)$ y teórico $(52.94 \%)$.

En tal sentido, se puede señalar que la naturaleza de la disciplina objeto de estudio de este trabajo exige un alto nivel de actividad y agilidad, así como soluciones prácticas y resolutivas, con un alto grado de actitud participante; sin embargo, también se debe apuntar que para todo esto se debe contar con una base teórica que permita entender y aplicar corrientes y modelos vigentes de manera correcta.

Igualmente, es necesario comentar, en cuanto a las limitaciones del estudio, que el cuestionario se aplicó en un solo momento, por lo que sería conveniente usarlo también durante toda la formación de los alumnos para observar y registrar si existen cambios en el transcurso de su proceso educativo.

Finalmente, la principal fortaleza de esta indagación radica en el aporte realizado a la literatura especializada sobre el tema en cuestión, pues se ha demostrado que los estilos de aprendizaje no necesariamente siguen una constante universal. En tal sentido, se deben tomar en cuenta distintas variables, como el país, el modo de vida y, por supuesto, las características propias de cada disciplina. En pocas palabras, en la educación de las personas no se puede partir de recetas establecidas debido a la complejidad de la esencia humana. 


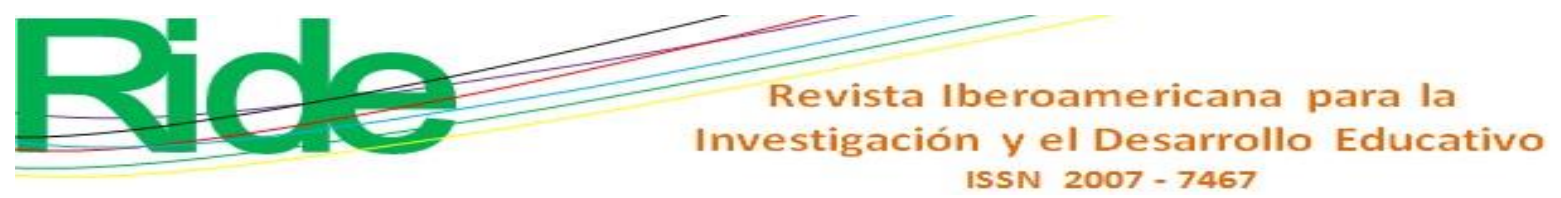

\section{Conclusiones}

De acuerdo con los diferentes autores citados en la introducción de este artículo, a nivel universitario los estudiantes deben de desarrollar el estilo de aprendizaje reflexivo; sin embargo, en el presente estudio se encontró lo contrario, pues el estilo que predomina en alumnos de la licenciatura en Educación Física y Ciencias del Deporte de la Universidad Autónoma de Querétaro es el activo. Aunado a esto, se puede afirmar que también existe una tendencia de los alumnos en cuanto a combinar estilos de aprendizaje (p. ej., activo-practico y activo-teórico), lo que se corresponde con las características de la carrera que estudian, donde se deben desempeñar actividades más prácticas y activas. Aun así, se debe advertir que el perfil laboral de este futuro profesional también se debe vincular con la atención de personas de diferentes grupos etarios con características específicas y/o con capacidades diferentes, los cuales requieren un análisis delimitado de sus necesidades y de las actividades físicas que deben desarrollar.

Ante esta situación, se propone realizar más investigaciones sobre el tema de estilos de aprendizaje y revisar las estrategias empeladas por los docentes en aulas para elaborar programas de intervención que permitan también potenciar el aprendizaje reflexivo; de ese modo se podrán ofrecer más herramientas para que los egresados se integren con mayor facilidad y de forma más eficiente al campo laboral. 


\section{Referencias}

Acevedo, D., Cavadla, S., y Alvis, A. (2015). Estilos de aprendizaje de los estudiantes de la Facultad de Ingeniería de la Universidad de Cartagena (Colombia). Formación Universitaria, 8(4), 15-22.

Alonso, C. M., Gallego, D. J. y Honey, P. (2007) Los estilos de aprendizaje. Procedimientos de diagnóstico y mejora (7. a ed). Bilbao. España: Ediciones Mensajero.

Camarero, F., Del Buey, F., y Herrero, J. (2000). Estilos y estrategias de aprendizaje en estudiantes universitarios. Psicothema, 12(4), 615-622.

Dunn, R., Dunn, K. And Price, G. (1985) Manual: Learning Style Inventory. Lawrence, KS: Price Systems.

Gil, P., Contreras, O., Pastor, J., Gómez, I., González, S., García, L., De Moya, M. y López, A. (2007). Estilos de aprendizaje de los estudiantes de magisterio: especial consideración de los alumnos de educación física. Revista de Currículum y Formación de Profesorado, 12(2), 1-19. Recuperado de http://www.redalyc.org/articulo.oa?id=56711209.

Isaza Valencia, L. (2014). Estilos de aprendizaje: una apuesta por el desempeño académico de los estudiantes en la educación superior. Revista Encuentros, 12(2), 25-34.

Keefe, J. (1988). Aprendiendo perfiles de aprendizaje: manual de examinador. Reston, Virginia: NASSP.

Salas-Cabrera, J. (2014). Estilos de aprendizaje en estudiantes de la Escuela de Ciencias del Movimiento Humano y Calidad de Vida. Revista Electrónica Educare, 18(3), 159171.

Serra-Olivares, Muñoz, C., Cejudo, C. y Gil, P. (2017). Estilos de aprendizaje y rendimiento académico de universitarios de Educación Física chilenos. Nuevas Tendencias en Educación Física, Deporte y Recreación, (32), 62-67. Recuperado de http:www.redalyc.org/articulo.oa?id=345751100013. 
Stenberg, R. (1997). Thinking Styles. Cambridge: Cambridge University Press.

\begin{tabular}{|c|c|}
\hline Rol de Contribución & Autor (es) \\
\hline Conceptualización & Beatriz Garza Gonzalez ( Principal) \\
\hline Metodología & $\begin{array}{l}\text { Beatriz Garza Gonzalez (Igual) Ma. Alejandra Hernández } \\
\text { Castañón (Igual) }\end{array}$ \\
\hline Software & No aplica \\
\hline Validación & $\begin{array}{l}\text { Oscar Angel Gómez Terán (principal) Sandra Jenny Cortés } \\
\text { Heredia (apoya) }\end{array}$ \\
\hline Análisis Formal & $\begin{array}{l}\text { Oscar Angel Gómez Terán( igual) Sandra Jenny Cortés } \\
\text { Heredia (igual) }\end{array}$ \\
\hline Investigación & $\begin{array}{l}\text { Oscar Angel Gómez Terán (igual) Sandra Jenny Cortés } \\
\text { Heredia (Igual) } \\
\text { Victorina Castrejon Reyes(Apoya) }\end{array}$ \\
\hline Recursos & $\begin{array}{l}\text { Ma. Alejandra Hernández Castañón (Igual) Victorina Castrejon } \\
\text { Reyes (igual) }\end{array}$ \\
\hline Curación de datos & $\begin{array}{l}\text { Oscar Angel Gómez Terán (Principal) Ma. Alejandra } \\
\text { Hernández Castañón (igual) }\end{array}$ \\
\hline $\begin{array}{l}\text { Escritura - Preparación del } \\
\text { borrador original }\end{array}$ & $\begin{array}{l}\text { Oscar Angel Gómez Terán (Principal) Sandra Jenny Cortés } \\
\text { Heredia (apoya) }\end{array}$ \\
\hline $\begin{array}{l}\text { Escritura - Revisión y } \\
\text { edición }\end{array}$ & $\begin{array}{l}\text { Oscar Angel Gómez Terán (Principal) Sandra Jenny Cortés } \\
\text { Heredia (apoya) } \\
\text { Beatriz Garza Gonzalez (apoya) }\end{array}$ \\
\hline Visualización & Oscar Angel Gómez Terán \\
\hline Supervisión & $\begin{array}{l}\text { Ma. Alejandra Hernández Castañón (Principal) Beatriz Garza } \\
\text { Gonzalez (apoya) }\end{array}$ \\
\hline $\begin{array}{l}\text { Administración de } \\
\text { Proyectos }\end{array}$ & $\begin{array}{l}\text { Ma. Alejandra Hernández Castañón (Principal) Beatriz Garza } \\
\text { Gonzalez (apoya) }\end{array}$ \\
\hline Adquisición de fondos & $\begin{array}{l}\text { Ma. Alejandra Hernández Castañón (Principal) Victorina } \\
\text { Castrejon Reyes (igual) }\end{array}$ \\
\hline
\end{tabular}

